\title{
Numerical modelling of transformation-induced damage and plasticity in metals
}

\author{
Akke S J Suiker and Sergio Turteltaub \\ Delft University of Technology, Faculty of Aerospace Engineering, P O Box 5058, \\ NL-2600 GB DELFT, The Netherlands \\ E-mail: a.s.j.suiker@tudelft.nl and s.r.turteltaub@tudelft.nl
}

Received 14 June 2006, in final form 19 September 2006

Published 7 December 2006

Online at stacks.iop.org/MSMSE/15/S147

\begin{abstract}
In multiphase carbon steels, irreversible martensitic transformations from a parent austenitic phase are often accompanied by plastic deformations in the surrounding phases and damage growth in the martensitic product phase. In the present communication the interactions between these complex processes are studied through three-dimensional numerical analyses, where the response of the austenitic phase is simulated using a phase-changing damage model and the response of the surrounding matrix is mimicked with a plasticity model. A numerical update algorithm is provided for the phase-changing damage model, which is based on a fully implicit backward Euler scheme formulated within the framework of finite deformations. The consistent tangent operator for the model is computed using a numerical differentiation technique. Special attention is given to the robust and accurate treatment of the various constraints related to the volume fractions and damaged volume fractions of the transformation parent and product phases. The numerical analyses of a multiphase carbon steel microstructure illustrate the effect of the transformation, plasticity and damage processes on the overall stress-strain response and on the transformation and damage evolutions of the sample for various austenite crystal orientations. The computed responses are in qualitative agreement with experimental results reported in the literature. In terms of the numerical model, a variation study of the mesh density shows that the numerical results tend to converge upon mesh refinement.
\end{abstract}

\section{Introduction}

Martensitic phase transformations play an important role in the thermomechanical performance of materials such as multiphase carbon steels, shape memory alloys and ceramics. The transition from an austenitic parent phase to a martensitic product phase occurs as a displacive 
transformation and results in a significant shape change of the microstructure. In multiphase carbon steels containing metastable austenite, this shape change may induce plasticity in the surrounding phases. Together with the development of a relatively hard martensitic product phase, this so-called 'transformation-induced plasticity (TRIP) effect' provides these steels with favourable strength and ductility properties (see for example, [1]). In ceramics such as zirconia, the shape change resulting from a martensitic phase transformation can delay or arrest crack propagation and thus is responsible for increasing the effective fracture toughness of the ceramic (see for example, [2]). In shape memory alloys the martensitic transformation is crystallographically reversible. Accordingly, these materials are furnished with a shape memory effect and a pseudo-elastic behaviour (see for example, [3]), which makes them particularly useful in specific functional applications, such as blood-clot filters, hydraulic couplings and force actuators.

In contrast to the beneficial properties mentioned above, martensitic transformations may also have a detrimental effect on the thermomechanical response of a material. This occurs if the martensitic product phase is relatively hard and brittle and provides nucleation sites for crystalline damage [4-9]. Since crystalline damage eventually may lead to crack formation at a higher length scale, it is important to understand the interplay between martensitic transformations and crystalline damage processes in detail. For this purpose, in a recent contribution of Suiker and Turteltaub [10] a thermomechanical model has been developed that describes the interactions between transformation and damage processes at the level of an austenitic grain. The constitutive parameters in the model are dependent on subgrain information following from the crystallographic theory of martensitic transformations [11,12]. For the specific phase transition of face-centred cubic (FCC) austenite to body-centred tetragonal (BCT) martensite, 24 martensitic transformation systems can be identified. The state of transformation in each of these transformation systems is represented by the corresponding volume fractions, while the state of crystalline damage is reflected by additional state variables, called the damaged volume fractions.

The present paper provides a time integration scheme for the phase-changing damage model developed in [10], where special attention is given to the robust, accurate treatment of the various constraints for the volume fractions and damaged volume fractions of the different phases. For the austenitic phase and the individual martensitic transformation systems these constraints reflect the activation and deactivation of transformation processes, damage processes and combinations thereof. It is one of the aims of this paper to demonstrate how this relatively complex set of constraints can be robustly implemented within a fully implicit stress-update algorithm, which is important for conducting reliable simulations on samples experiencing complex microstructural changes due to crystalline damage and transformation processes. The update algorithm is based upon a backward Euler scheme and is formulated within the framework of finite deformations. The computation of the consistent tangent operator is carried out using a forward finite-difference scheme. The robustness of the numerical implementation is demonstrated through solving various three-dimensional boundary value problems of a grain of retained austenite embedded in ferritic matrix. This geometry represents the phases of a TRIP steel microstructure, where the transformation of retained austenite induces damage in the brittle martensitic product phase and plasticity in the surrounding ferritic matrix [7,9]. In order to illustrate the influence of crystalline martensitic damage on the overall response of the sample, for different crystal orientations simulations where damage in the martensite is allowed are compared with simulations where damage in the martensite is prevented. These simulations clearly show that the development of crystalline damage limits the development of stress in the martensitic product phase, where the value of the maximum stress is dependent of the grain orientation. Furthermore, the effect of the 


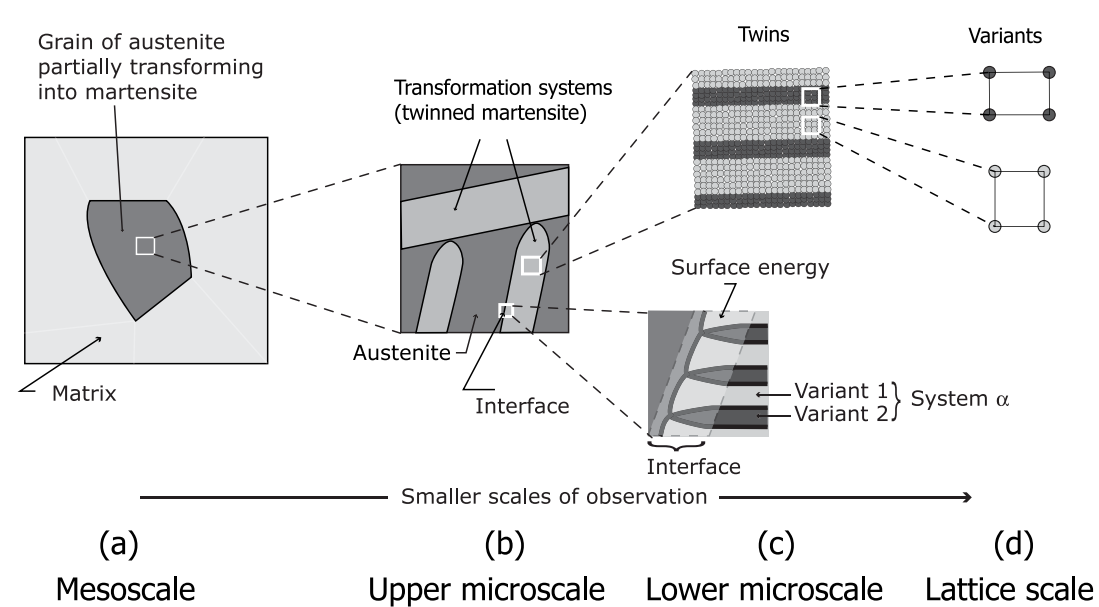

Figure 1. Martensitic substructures at four hiarchial scales of observation.

transformation rate on the hardening capacity of the TRIP steel microstructure is demonstrated, which shows a trend that is in correspondence with experimental observations. Finally, the convergence behaviour of the numerical response is analysed through performing a mesh refinement study.

\section{Summary of model equations}

In this section the main equations of the phase-changing damage model presented in Suiker and Turteltaub [10] are summarized. Since in the present communication attention is restricted to the numerical analysis of isothermal processes, only the mechanical part of the model is considered. The complete thermomechanical case can be treated through an extension of the algorithm presented in this paper. The phase-changing damage model can be used to simulate the constitutive response of an austenitic phase in metals. The material structures considered in the model are schematized in figure 1, where four hiarchial scales of observation are distinguished: (i) the mesoscale, (ii) the upper microscale, (iii) the lower microscale and (iv) the lattice scale. At the mesoscale, regions of martensite can develop inside the grain of retained austenite upon mechanical and/or thermal loading (figure 1(a)). The microstructural shape changes caused by the martensitic transformations may generate plastic deformations in the surrounding matrix, which in the present study are accounted for using a separate plasticity model. The plastic deformations in the austenite are assumed to remain small and are therefore neglected in the phase-changing damage model. This assumption is reasonable for TRIP steels with a relatively high carbon concentration in the austenite (i.e. $>1.4 \mathrm{wt} . \%$ ). The martensite is thus supposed to be formed by a so-called stress-assisted transformation, rather than by a so-called strain-induced transformation that is characterized by plastic deformations in the austenitic parent phase. At the upper microscale, the martensite appears as plates with a specific orientation, which are referred to as martensitic transformation systems (figure $1(b)$ ). Further magnification to the lower microscale shows that each martensitic plate is composed of a layered, twin-related structure of two martensitic variants (figure 1(c)). At the interface between the martensite and the austenite, which is commonly called the habit plane, the coherent connection of the lattice structures of both phases introduces a local deformation field. The strain energy corresponding to this local deformation field can be interpreted as a 
surface energy. At the lattice scale the martensite within a twin layer appears as one out of three possible variants that have a characteristic tetragonal lattice structure (figure $1(d)$ ).

At the mesoscale, the effective deformation gradient $\boldsymbol{F}$ in a material point within a grain of retained austenite is decomposed as

$$
\boldsymbol{F}=\boldsymbol{F}_{\mathrm{e}} \boldsymbol{F}_{\text {tr }},
$$

where $\boldsymbol{F}_{\mathrm{e}}$ and $\boldsymbol{F}_{\text {tr }}$ are the elastic and transformation deformation gradients, respectively. The transformation deformation gradient $\boldsymbol{F}_{\text {tr }}$ maps a material point from the (undeformed) reference configuration to a (stress-free) intermediate configuration, whereas the elastic deformation gradient $\boldsymbol{F}_{\mathrm{e}}$ maps a material point from the intermediate configuration to the current configuration. The multiplicative decomposition of the total deformation gradient is similar to that used in large deformation plasticity models $[13,14]$, where $\boldsymbol{F}_{\text {tr }}$ plays the role of the plastic deformation gradient $\boldsymbol{F}_{\mathrm{p}}$. The transformation deformation gradient can be expressed as [15]

$$
\boldsymbol{F}_{\mathrm{tr}}=\boldsymbol{I}+\sum_{\alpha=1}^{N} \xi^{(\alpha)} \boldsymbol{\gamma}^{(\boldsymbol{\alpha})}
$$

Here, $\xi^{(\alpha)}$ is the volume fraction of a martensitic transformation system $\alpha$, measured in the undeformed reference configuration, and $\boldsymbol{I}$ is the second-order identity tensor. The martensitic transformation systems range from $\alpha=1,2, \ldots, N$, where $N$ represents the total number of transformation systems, which, for a transformation from FCC austenite to BCT martensite, is equal to $24[11,16]$. Furthermore, the parameter $\gamma^{(\alpha)}$ appearing in equation (2) is the transformation strain, given by

$$
\gamma^{(\alpha)}:=\gamma_{\mathrm{T}} \hat{\boldsymbol{b}}^{(\alpha)} \otimes \boldsymbol{m}^{(\alpha)}
$$

where $\boldsymbol{m}^{(\alpha)}$ is the unit vector normal to the habit plane of the transformation system and $\hat{\boldsymbol{b}}^{(\alpha)}=\boldsymbol{b}^{(\alpha)} / \gamma_{\mathrm{T}}$ is the normalized shape strain vector. The habit plane vectors can be computed from the theory of martensitic transformations $[3,11,12]$ and serve as input for the present model. The shape strain magnitude is equal to $\gamma_{\mathrm{T}}=\left\|\boldsymbol{b}^{(\alpha)}\right\|$ and is identical for all transformation systems $\alpha$. The stress-strain relation used in the model is

$$
\boldsymbol{S}=\mathbb{C}_{\mathrm{d}} \boldsymbol{E}_{\mathrm{e}},
$$

where $S$ is the second Piola-Kirchhoff stress in the intermediate configuration, $\mathbb{C}_{\mathrm{d}}$ is the effective stiffness and $\boldsymbol{E}_{\mathrm{e}}=\left(\boldsymbol{F}_{\mathrm{e}}^{\mathrm{T}} \boldsymbol{F}_{\mathrm{e}}-\boldsymbol{I}\right) / 2$ is the elastic Green-Lagrange strain. The stiffness tensor $\mathbb{C}_{\mathrm{d}}$ is dependent of the transformation and damage properties of the material and has the form

$$
\begin{aligned}
\mathbb{C}_{\mathrm{d}}=\hat{\mathbb{C}}_{\mathrm{d}}\left(\boldsymbol{\xi}, \boldsymbol{\xi}_{\mathrm{d}}\right) & =\frac{1}{\operatorname{det}\left(\boldsymbol{F}_{\mathrm{tr}}(\boldsymbol{\xi})\right)}\left\{\left(\left(1-\sum_{\alpha=1}^{N} \xi^{(\alpha)}\right)-\xi_{\mathrm{d}}^{(0)}\right) \mathbb{C}^{\mathrm{A}}\right. \\
& \left.+\left(1+\delta_{\mathrm{T}}\right) \sum_{\alpha=1}^{N}\left(\xi^{(\alpha)}-\xi_{\mathrm{d}}^{(\alpha)}\right) \mathbb{C}^{(\alpha)}\right\},
\end{aligned}
$$

where $\mathbb{C}^{\mathrm{A}}$ and $\mathbb{C}^{(\alpha)}$ are stiffness tensors containing the elastic properties of FCC austenite and the twinned BCT martensite appearing in transformation system $\alpha$, respectively. The parameter $\delta_{\mathrm{T}}$ represents the volumetric change due to transformation and is given by

$$
\delta_{\mathrm{T}}:=\gamma_{\mathrm{T}} \hat{\boldsymbol{b}}^{(\alpha)} \cdot \boldsymbol{m}^{(\alpha)}, \quad \forall \alpha=1,2, \ldots, N .
$$

In addition, $\boldsymbol{\xi}_{\mathrm{d}}:=\left\{\xi_{\mathrm{d}}^{(0)}, \xi_{\mathrm{d}}^{(1)}, \ldots, \xi_{\mathrm{d}}^{(N)}\right\}$ represents the vector of damaged volume fractions, with $\xi_{\mathrm{d}}^{(0)}$ the damaged volume fraction of austenite and $\xi_{\mathrm{d}}^{(\alpha)}$ the damaged volume fraction of 


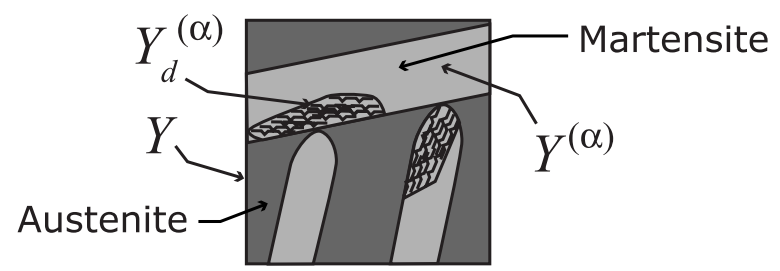

Figure 2. Schematization at the upper microscale of a damaged martensitic region $Y_{\mathrm{d}}^{(\alpha)}$. The parameters $Y^{(\alpha)}$ and $Y$ indicate the total region of the martensitic transformation system $\alpha$ and the total region of the microstructure, respectively. The above figure can be included in the multiscale representation in figure 1 by replacing figure $1(b)$.

a martensitic transformation system $\alpha=1,2, \ldots, N$. In figure 2 the generation of damage within the martensite is schematized at the upper microscale. This figure can be included in the multiscale representation in figure 1 by replacing figure $1(b)$. In accordance with figure 2, the volume fraction $\xi^{(\alpha)}$ and the damaged volume fraction $\xi_{\mathrm{d}}^{(\alpha)}$ can be expressed as

$$
\xi^{(\alpha)}:=\frac{\left|Y^{(\alpha)}\right|}{|Y|}, \quad \xi_{\mathrm{d}}^{(\alpha)}:=\frac{\left|Y_{\mathrm{d}}^{(\alpha)}\right|}{|Y|}
$$

where $Y^{(\alpha)}$ is the total region of the martensitic transformation system $\alpha, Y_{\mathrm{d}}^{(\alpha)}$ is the damaged region of the martensitic transformation system $\alpha$ and $Y$ is the total region of the microstructure at the upper microscale. The volume fractions and damaged volume fractions are bounded by the constraints

$0 \leqslant \xi_{\mathrm{d}}^{(0)}+\sum_{\alpha=1}^{N} \xi^{(\alpha)} \leqslant 1, \quad 0 \leqslant \xi_{\mathrm{d}}^{(\alpha)} \leqslant \xi^{(\alpha)} \leqslant 1, \quad 1-\sum_{\alpha=1}^{N} \xi^{(\alpha)}=\xi^{(0)}$

with $\xi^{(0)}$ representing the volume fraction of the austenite.

The kinetic law describing the transformation process in a specific martensitic transformation system $\alpha$ is expressed as

$$
\dot{\xi}^{(\alpha)}= \begin{cases}\dot{\xi}_{\max }^{(\alpha)} \tanh \left(\frac{1}{v^{(\alpha)}} \frac{\left(f^{(\alpha)}-f_{\mathrm{cr}}^{(\alpha)}\right)}{f_{\mathrm{cr}}^{(\alpha)}}\right) & \text { for } f^{(\alpha)}>f_{\mathrm{cr}}^{(\alpha)} \\ 0 & \text { for } f^{(\alpha)} \leqslant f_{\mathrm{cr}}^{(\alpha)}\end{cases}
$$

with $\dot{\xi}_{\text {max }}^{(\alpha)}$ the maximum transformation rate, $v^{(\alpha)}$ a transformation-related viscosity parameter, $f^{(\alpha)}$ the driving force for transformation and $f_{\mathrm{cr}}^{\alpha}$ the critical value for the transformation driving force. As shown in equation (9), the transformation process is activated when the transformation driving force of one or more transformations systems exceeds the critical transformation threshold, $f^{(\alpha)}>f_{\mathrm{cr}}^{(\alpha)}$. The transformation rates in equation (9) are prescribed to be non-negative, $\dot{\xi}^{(\alpha)} \geqslant 0$, such that the transformation process is irreversible, i.e. the martensite cannot transform back into austenite. This type of martensitic transformation is representative of the retained austenite phase present in TRIP steels. 
The kinetic law describing the development of crystalline damage in a transformation system is formulated as ${ }^{1}$

$\dot{\xi}_{\mathrm{d}}^{(\alpha)}=\left\{\begin{array}{lc}\dot{\xi}_{\mathrm{d}, \max }^{(\alpha)}\left(\left(1-\exp \left(-\beta_{\mathrm{d}}^{(\alpha)} \frac{\left(g^{(\alpha)}-g_{\mathrm{cr}}^{(\alpha)}\right)}{g_{\mathrm{cr}}^{(\alpha)}}\right)\right)\left(\xi^{(\alpha)}-\xi_{\mathrm{d}}^{(\alpha)}\right)\right) & \text { for } g^{(\alpha)}>g_{\mathrm{cr}}^{(\alpha)} \\ 0 & \text { for } g^{(\alpha)} \leqslant g_{\mathrm{cr}}^{(\alpha)}\end{array}\right.$.

Here, the parameter $\dot{\xi}_{\mathrm{d}, \max }^{(\alpha)}$ represents the maximum damage rate and $\beta_{\mathrm{d}}^{(\alpha)}$ couples the damage growth to the value of the damage driving force $g^{(\alpha)}$. It can be noticed that damage is activated if the damage driving force exceeds a critical value, $g^{(\alpha)}>g_{\mathrm{cr}}^{(\alpha)}$. In accordance with the kinetic law, equation (10), damage is considered to be an irreversible process, i.e. $\dot{\xi}_{\mathrm{d}}^{(\alpha)} \geqslant 0$. Further, the specific dependence of the damage kinetic law on $\xi^{(\alpha)}$ and $\xi_{\mathrm{d}}^{(\alpha)}$ warrants that the damaged volume fraction $\xi_{\mathrm{d}}^{(\alpha)}$ asymptotically approaches the corresponding volume fraction $\xi^{(\alpha)}$ for any value of $g^{(\alpha)}>g_{\mathrm{cr}}^{(\alpha)}$ (under the assumption of $\beta_{\mathrm{d}}^{(\alpha)}>0, \dot{\xi}_{\mathrm{d}, \max }^{(\alpha)}>0$ ). Consequently, the requirement $\xi_{\mathrm{d}}^{(\alpha)} \leqslant \xi^{(\alpha)}$, which has been stated previously in equation $(8)_{2}$, is unconditionally satisfied. In the limit case of $\dot{\xi}_{\mathrm{d}, \max }^{(\alpha)} \rightarrow \infty$, the rate-dependent kinetic law, equation (10), turns into a rate-independent damage formulation that can be expressed in terms of the loading function $F^{(\alpha)}$ as

$F^{(\alpha)}=\hat{F}^{(\alpha)}\left(\xi^{(\alpha)}, \xi_{\mathrm{d}}^{(\alpha)}\right)=\xi^{(\alpha)}-\xi_{\mathrm{d}}^{(\alpha)}=0 \quad$ for $\quad g^{(\alpha)}>g_{\mathrm{cr}}^{(\alpha)}$,

where the loading and unloading conditions are prescribed by the following Kuhn-Tucker conditions:

$F^{(\alpha)} \dot{\xi}_{\mathrm{d}}^{(\alpha)}=0, \quad F^{(\alpha)} \leqslant 0, \quad \dot{\xi}_{\mathrm{d}}^{(\alpha)} \geqslant 0 \quad$ with $\quad \alpha=0,1, \ldots, N$.

The rate-independent model, equation (11), reflects the ideally brittle case with complete damage occurring instantaneously in a material point as soon as the damage driving force $g^{(\alpha)}$ exceeds the critical value $g_{\mathrm{cr}}^{(\alpha)}$. Such a formulation is analogous to Griffiths' fracture criterion for an ideally brittle interfacial crack.

In the present study, the kinetic laws for transformation and damage are taken as ratedependent, in agreement with equations (9) and (10). In addition to the physical arguments leading to this choice $[10,15]$, in comparison with rate-independent kinetic laws, ratedependent kinetic laws have the advantage that they may regularize the mechanical response. Consequently, bifurcations in the numerical procedure (e.g. due to a non-uniqueness of the actual set of transformation systems activated) can be prevented.

The final ingredients to the model are the driving forces for transformation and crystalline damage, $f^{(\alpha)}$ and $g^{(\alpha)}$. These driving forces are derived from a specific form of the Helmholtz energy that is consistent with the constitutive relation given by equations (4) and (5). Accordingly, the following expressions for the driving forces are obtained

$$
\begin{array}{lll}
f^{(\alpha)}=f_{\mathrm{m}}^{(\alpha)}+f_{\mathrm{th}}^{(\alpha)}+f_{\mathrm{s}}^{(\alpha)} & \text { with } & \alpha=1,2, \ldots, N, \\
g^{(\alpha)}=g_{\mathrm{m}}^{(\alpha)}+g_{\mathrm{th}}^{(\alpha)}+g_{\mathrm{s}}^{(\alpha)} & \text { with } & \alpha=0,1, \ldots, N,
\end{array}
$$

where $f_{\mathrm{m}}^{(\alpha)}$ are $g_{\mathrm{m}}^{(\alpha)}$ are the mechanical driving forces $f_{\mathrm{th}}^{(\alpha)}$ and $g_{\mathrm{th}}^{(\alpha)}$ are the thermal driving forces and $f_{\mathrm{s}}^{(\alpha)}$ and $g_{\mathrm{s}}^{(\alpha)}$ are driving force components related to the surface energy at the

1 The kinetic law, equation (10), is obtained from a slight modification of the damage kinetic law proposed in [10], i.e. the term $(1-\exp (.).) \xi^{(\alpha)}-\xi_{\mathrm{d}}^{(\alpha)}$ of the kinetic law in [10] is replaced by $(1-\exp (.)).\left(\xi^{(\alpha)}-\xi_{\mathrm{d}}^{(\alpha)}\right)$. The reason for this modification is that the form given by equation (10) provides a better overall convergence behaviour of the numerical boundary value problems studied in section 4 than the kinetic law proposed in [10]. Nonetheless, using the model parameter values presented in table 1, additional computations (not presented here) for an individual material point subjected to uniaxial tension show that the two kinetic laws lead to virtually the same numerical result. 
transformation habit planes (see also figure $1(c)$ ), in accordance with

$$
\begin{aligned}
& f_{\mathrm{m}}^{(\alpha)}=\operatorname{det}\left(\boldsymbol{F}_{\mathrm{tr}}\right) \boldsymbol{F}_{\mathrm{e}}^{T} \boldsymbol{F}_{\mathrm{e}} \boldsymbol{S} \boldsymbol{F}_{\mathrm{tr}}^{-T} \cdot \gamma^{(\alpha)}+\frac{1}{2}\left(\mathbb{C}^{\mathrm{A}}-\left(1+\delta_{\mathrm{T}}\right) \mathbb{C}^{(\alpha)}\right) \boldsymbol{E}_{\mathrm{e}} \cdot \boldsymbol{E}_{\mathrm{e}}, \\
& f_{\mathrm{s}}^{(\alpha)}=-\frac{\chi}{l_{0}}\left(1-2\left(\xi^{(\alpha)}-\xi_{\mathrm{d}}^{(\alpha)}\right)\right), \\
& g_{\mathrm{m}}^{(\alpha)}= \begin{cases}\frac{1}{2} \mathbb{C}^{\mathrm{A}} \boldsymbol{E}_{\mathrm{e}} \cdot \boldsymbol{E}_{\mathrm{e}} & \text { for } \alpha=0, \\
\frac{1}{2}\left(1+\delta_{\mathrm{T}}\right) \mathbb{C}^{(\alpha)} \boldsymbol{E}_{\mathrm{e}} \cdot \boldsymbol{E}_{\mathrm{e}} & \text { for } \alpha=1,2, \ldots, N,\end{cases} \\
& g_{\mathrm{s}}^{(\alpha)}= \begin{cases}0 & \text { for } \alpha=0, \\
\frac{\chi}{l_{0}}\left(1-2\left(\xi^{(\alpha)}-\xi_{\mathrm{d}}^{(\alpha)}\right)\right) & \text { for } \alpha=1,2, \ldots, N .\end{cases}
\end{aligned}
$$

Expressions for the thermal driving forces for transformation, $f_{\mathrm{th}}^{(\alpha)}$, and damage, $g_{\mathrm{th}}^{(\alpha)}$, can be found in [10]. The present work focuses on isothermal analyses, for which the thermal driving forces $f_{\mathrm{th}}^{(\alpha)}$ and $g_{\mathrm{th}}^{(\alpha)}$ are constant and identical for all transformation systems $\alpha$. Observe from equation (14) that the mechanical driving force for transformation, $f_{\mathrm{m}}^{(\alpha)}$, is composed of a 'resolved stress' contribution and a contribution related to the stiffness mismatch between austenite and martensite. Furthermore, the damage driving force component, $g_{\mathrm{m}}^{(\alpha)}$, equals (i) the elastic bulk energy of the undamaged austenite if $\alpha=0$ and (ii) the bulk energy of the undamaged martensite if $\alpha=1,2, \ldots, N$. Accordingly, this driving force can be interpreted as the bulk energy release rate per unit advance of damaged volume of the corresponding phase. The surface energy contributions to the transformation and damage processes, $f_{\mathrm{s}}^{(\alpha)}$ and $g_{\mathrm{s}}^{(\alpha)}$, are set by an interfacial energy per unit area $\chi$ at the transformation habit planes and a length scale parameter $l_{0}$ denoting the geometry of a plate of twinned martensite inside a grain of retained austenite, measured when the transformation nucleates (see also [15]).

\section{Numerical integration procedure}

This section addresses the numerical integration procedure of the phase-changing damage model. The update algorithm presented here has been implemented as a 'user subroutine' in the finite element program ABAQUS. The time integration procedure is based on a fully implicit backward Euler algorithm, in which during a time interval $\left[t_{n}, t_{n+1}\right]$ the primary variables of the model are evaluated at time $t_{n+1}$, assuming their values at time $t_{n}$ are known. In a displacementbased finite element method, the update of the primary variables within a large deformation setting is driven by the incremental total deformation gradient

$$
\Delta \boldsymbol{F}_{n+1}:=\boldsymbol{F}_{n+1}-\boldsymbol{F}_{n},
$$

with $\boldsymbol{F}_{n}$ and $\boldsymbol{F}_{n+1}$ the deformation gradients at time steps $t_{n}$ and $t_{n+1}$, respectively.

\subsection{Primary variables and residual equations}

The primary variables are assembled in a vector $\boldsymbol{a}$. The essential equations related to these primary variables are expressed in a residual format, and assembled in a vector $\boldsymbol{r}$. The updated vector of primary variables $\boldsymbol{a}_{n+1}$ is then obtained solving the system of residual equations $\boldsymbol{r}_{n+1}$ within a prescribed tolerance (see also [17-19]), i.e.

$$
\boldsymbol{r}_{n+1}:=\check{\boldsymbol{r}}\left(\boldsymbol{a}_{n+1}\right)=\mathbf{0} .
$$

Since the equations assembled in $\boldsymbol{r}_{n+1}$ generally are coupled and nonlinear, the update $\boldsymbol{a}_{n+1}$ needs to be established in an iterative fashion. This is done using a Newton-Raphson procedure, 
where the vector of primary variables at the present iteration, $\boldsymbol{a}_{n+1}^{k+1}$, is determined from the vector of residuals at the previous iteration, $\boldsymbol{r}_{n+1}^{k}$, and the vector of primary variables at the previous iteration, $\boldsymbol{a}_{n+1}^{k}$, i.e.

$$
\boldsymbol{a}_{n+1}^{k+1}=\boldsymbol{a}_{n+1}^{k}-\left(\frac{\mathrm{d} \check{\boldsymbol{r}}_{n+1}^{k}}{\mathrm{~d} \boldsymbol{a}_{n+1}^{k}}\right)^{-1} \boldsymbol{r}_{n+1}^{k} .
$$

In the phase-changing damage model the vector of primary variables is composed of four categories, namely $\boldsymbol{a}_{n+1}=\left[\boldsymbol{a}_{n+1}^{\mathrm{I}}, \boldsymbol{a}_{n+1}^{\mathrm{II}}, \boldsymbol{a}_{n+1}^{\mathrm{III}}, \boldsymbol{a}_{n+1}^{\mathrm{IV}},\right]^{\mathrm{T}}$. These categories are given by

$$
\begin{aligned}
& \boldsymbol{a}_{n+1}^{\mathrm{I}}:=\Delta \boldsymbol{\xi}_{n+1}, \\
& \boldsymbol{a}_{n+1}^{\mathrm{II}}:=\Delta \boldsymbol{\xi}_{\mathrm{d}, n+1}, \\
& \boldsymbol{a}_{n+1}^{\mathrm{III}}:=\left[\mathbb{C}_{\mathrm{d}, n+1}\right], \\
& \boldsymbol{a}_{n+1}^{\mathrm{IV}}:=\left[\boldsymbol{S}_{n+1}\right],
\end{aligned}
$$

where $\Delta \boldsymbol{\xi}_{n+1}$ are the incremental volume fractions, $\Delta \boldsymbol{\xi}_{\mathrm{d}, n+1}$ are the incremental damaged volume fractions, $\mathbb{C}_{\mathrm{d}, n+1}$ is the effective stiffness tensor and $\boldsymbol{S}_{n+1}$ is the second Piola-Kirchhoff stress, measured in the intermediate configuration. In equation (18) the square brackets [...] indicate that the actual object, which is a tensor of the second or the fourth order, is represented as a vector.

In correspondence with the above-mentioned four categories that construct the vector of primary variables, the four categories of the vector of residuals, $\boldsymbol{r}_{n+1}=\left[\boldsymbol{r}_{n+1}^{\mathrm{I}}, \boldsymbol{r}_{n+1}^{\mathrm{II}}, \boldsymbol{r}_{n+1}^{\mathrm{III}}, \boldsymbol{r}_{n+1}^{\mathrm{IV}}\right]^{\mathrm{T}}$, are

$$
\begin{aligned}
\boldsymbol{r}_{n+1}^{\mathrm{I}} & :=\Delta \boldsymbol{\xi}_{n+1}-\boldsymbol{\zeta}_{n+1} \Delta t_{n+1}, \\
\boldsymbol{r}_{n+1}^{\mathrm{II}} & :=\Delta \boldsymbol{\xi}_{\mathrm{d}, n+1}-\boldsymbol{\zeta}_{\mathrm{d}, n+1} \Delta t_{n+1}, \\
\boldsymbol{r}_{n+1}^{\mathrm{III}} & :=\left[\mathbb{C}_{\mathrm{d}, n+1}-\frac{1}{\operatorname{det}\left(\boldsymbol{F}_{\mathrm{tr}, n+1}\right)}\left\{\left(\left(1-\sum_{\alpha=1}^{N} \xi_{n+1}^{(\alpha)}\right)-\xi_{\mathrm{d}, n+1}^{(0)}\right) \mathbb{C}^{\mathrm{A}}\right.\right. \\
+ & \left.\left.\left(1+\delta_{\mathrm{T}}\right) \sum_{\alpha=1}^{N}\left(\xi_{n+1}^{(\alpha)}-\xi_{\mathrm{d}, n+1}^{(\alpha)}\right) \mathbb{C}^{(\alpha)}\right\}\right], \\
\boldsymbol{r}_{n+1}^{\mathrm{IV}} & :=\left[\boldsymbol{S}_{n+1}-\mathbb{C}_{\mathrm{d}, n+1} \boldsymbol{E}_{\mathrm{e}, n+1}\right],
\end{aligned}
$$

with the components of the auxiliary vectors $\boldsymbol{\zeta}_{n+1}$ and $\boldsymbol{\zeta}_{\mathrm{d}, n+1}$ as

$$
\zeta_{n+1}^{(\alpha)}= \begin{cases}\dot{\xi}_{\max }^{(\alpha)} \tanh \left(\frac{1}{v^{(\alpha)}} \frac{\left(f_{n+1}^{(\alpha)}-f_{\mathrm{cr}}^{(\alpha)}\right)}{f_{\mathrm{cr}}^{(\alpha)}}\right) & \text { for } \quad f_{n+1}^{(\alpha)}>f_{\mathrm{cr}}^{(\alpha)} \\ 0 & \text { for } \quad f_{n+1}^{(\alpha)} \leqslant f_{\mathrm{cr}}^{(\alpha)}\end{cases}
$$

and

$$
=\left\{\begin{array}{ll}
\dot{\xi}_{\mathrm{d}, \max }^{(\alpha)}\left(\left(1-\exp \left(-\beta_{\mathrm{d}}^{(\alpha)} \frac{\left(g_{n+1}^{(\alpha)}-g_{\mathrm{cr}}^{(\alpha)}\right)}{g_{\mathrm{cr}}^{(\alpha)}}\right)\right)\left(\xi_{n+1}^{(\alpha)}-\xi_{\mathrm{d}, n+1}^{(\alpha)}\right)\right) & \text { for } g_{n+1}^{(\alpha)}>g_{\mathrm{cr}}^{(\alpha)} \\
0 & \text { for } g_{n+1}^{(\alpha)} \leqslant g_{\mathrm{cr}}^{(\alpha)}
\end{array} .\right.
$$

The first and second residuals in equation (19) have been discretized in accordance with the implicit backward Euler method, i.e. $\Delta \boldsymbol{\xi}_{n+1} \approx \dot{\boldsymbol{\xi}}_{n+1} \Delta t_{n+1}$ and $\Delta \boldsymbol{\xi}_{\mathrm{d}, n+1} \approx \dot{\boldsymbol{\xi}}_{\mathrm{d}, n+1} \Delta t_{n+1}$, respectively, followed by substitution of the kinetic laws, equations (9) and (10), into these expressions. The third residual in equation (19) represents the update of the effective stiffness 
tensor, equation (5), and the fourth residual reflects the update of the stress, in correspondence with the constitutive relation, equation (4). The kinetic laws in equations (20) and (21) involve the driving forces $f_{n+1}^{(\alpha)}$ and $g_{n+1}^{(\alpha)}$, which are obtained from evaluating equations (13) and (14) at $t_{n+1}$, i.e.

$f_{n+1}^{(\alpha)}=f_{\mathrm{m}, n+1}^{(\alpha)}+f_{\mathrm{th}}^{(\alpha)}+f_{\mathrm{s}, n+1}^{(\alpha)} \quad$ with $\alpha=1,2, \ldots, N$,
$g_{n+1}^{(\alpha)}=g_{\mathrm{m}, n+1}^{(\alpha)}+g_{\mathrm{th}}^{(\alpha)}+g_{\mathrm{s}, n+1}^{(\alpha)}$$\quad$ with $\alpha=0,1, \ldots, N$,

where

$$
\begin{aligned}
& f_{\mathrm{m}, n+1}^{(\alpha)}= \operatorname{det}\left(\boldsymbol{F}_{\mathrm{tr}, n+1}\right) \boldsymbol{F}_{\mathrm{e}, n+1}^{T} \boldsymbol{F}_{\mathrm{e}, n+1} \boldsymbol{S}_{n+1} \boldsymbol{F}_{\mathrm{tr}, n+1}^{-T} \cdot \gamma^{(\alpha)} \\
&+\frac{1}{2}\left(\mathbb{C}^{\mathrm{A}}-\left(1+\delta_{\mathrm{T}}\right) \mathbb{C}^{(\alpha)}\right) \boldsymbol{E}_{\mathrm{e}, n+1} \cdot \boldsymbol{E}_{\mathrm{e}, n+1}, \\
& f_{\mathrm{s}, n+1}^{(\alpha)}=-\frac{\chi}{l_{0}}\left(1-2\left(\xi_{n+1}^{(\alpha)}-\xi_{\mathrm{d}, n+1}^{(\alpha)}\right)\right), \text { for } \alpha=0, \\
& g_{\mathrm{m}, n+1}^{(\alpha)}= \begin{cases}\frac{1}{2} \mathbb{C}^{\mathrm{A}} \boldsymbol{E}_{\mathrm{e}, n+1} \cdot \boldsymbol{E}_{\mathrm{e}, n+1} & \text { for } \alpha=1,2, \ldots, N, \\
\frac{1}{2}\left(1+\delta_{\mathrm{T}}\right) \mathbb{C}^{(\alpha)} \boldsymbol{E}_{\mathrm{e}, n+1} \cdot \boldsymbol{E}_{\mathrm{e}, n+1} & \end{cases}
\end{aligned}
$$

$g_{\mathrm{s}, n+1}^{(\alpha)}= \begin{cases}0 & \text { for } \alpha=0, \\ \frac{\chi}{l_{0}}\left(1-2\left(\xi_{n+1}^{(\alpha)}-\xi_{\mathrm{d}, n+1}^{(\alpha)}\right)\right) & \text { for } \alpha=1,2, \ldots, N .\end{cases}$

Under isothermal conditions the thermal contributions to the transformation driving force $f_{\mathrm{th}}^{(\alpha)}$ and the damage driving force $g_{\mathrm{th}}^{(\alpha)}$ are constant and are computed by substituting the ambient temperature of the modelled sample into the expressions for $f_{\text {th }}^{(\alpha)}$ and $g_{\text {th }}^{(\alpha)}$, see [10]. Furthermore, the variables $\boldsymbol{F}_{\mathrm{tr}, n+1}, \boldsymbol{F}_{\mathrm{e}, n+1}, \boldsymbol{E}_{\mathrm{e}, n+1}, \boldsymbol{\xi}_{n+1}$ and $\boldsymbol{\xi}_{\mathrm{d}, n+1}$ appearing in equations (19) and (23) are computed as

$$
\begin{aligned}
& \boldsymbol{F}_{\mathrm{tr}, n+1}=\boldsymbol{F}_{\mathrm{tr}, n}+\Delta \boldsymbol{F}_{\mathrm{tr}, n+1} \quad \text { where } \Delta \boldsymbol{F}_{\mathrm{tr}, n+1}=\sum_{\alpha=1}^{N} \Delta \xi_{n+1}^{(\alpha)} \gamma^{(\boldsymbol{\alpha})}, \\
& \boldsymbol{F}_{\mathrm{e}, n+1}=\boldsymbol{F}_{n+1} \boldsymbol{F}_{\mathrm{tr}, n+1}^{-1}, \\
& \boldsymbol{E}_{\mathrm{e}, n+1}=\frac{1}{2}\left(\boldsymbol{F}_{\mathrm{e}, n+1}^{\mathrm{T}} \boldsymbol{F}_{\mathrm{e}, n+1}-\boldsymbol{I}\right), \\
& \boldsymbol{\xi}_{n+1}=\boldsymbol{\xi}_{n}+\Delta \boldsymbol{\xi}_{n+1}, \\
& \boldsymbol{\xi}_{\mathrm{d}, n+1}=\boldsymbol{\xi}_{\mathrm{d}, n}+\Delta \boldsymbol{\xi}_{\mathrm{d}, n+1} .
\end{aligned}
$$

Observe that $\boldsymbol{F}_{\mathrm{e}, n+1}$ is obtained through inverting the decomposition, equation (1). The expression for $\Delta \boldsymbol{F}_{\mathrm{tr}, n+1}$ follows from taking the rate form of equation (2) and discretizing the result with respect to time.

\subsection{Trial state}

The iterative procedure presented by equation (17) is initiated using an elastic trial state, which is obtained by initially freezing the internal state variables in equations (19)-(24) to their known values at the previous time step $t_{n}$. For the present model, the trial state can be formally expressed as

$$
\left\{\boldsymbol{F}_{\mathrm{tr}, n+1}^{\mathrm{trial}}, \boldsymbol{\xi}_{n+1}^{\text {trial }}, \boldsymbol{\xi}_{\mathrm{d}, n+1}^{\mathrm{trial}}\right\}:=\left\{\boldsymbol{F}_{\mathrm{tr}, n}, \boldsymbol{\xi}_{n}, \boldsymbol{\xi}_{\mathrm{d}, n}\right\},
$$


which implies that at the onset of the Newton-Raphson procedure the incremental state variables are given by $\left\{\Delta \boldsymbol{F}_{\mathrm{tr}, n+1}, \Delta \boldsymbol{\xi}_{n+1}, \Delta \boldsymbol{\xi}_{\mathrm{d}, n+1}\right\}:=\{\mathbf{0}, \mathbf{0}, \mathbf{0}\}$. If the material state at time $t_{n}$ relates to a fully austenitic material with no damage, the trial state, equation (25), reduces to

$$
\left\{\boldsymbol{F}_{\mathrm{tr}, n+1}^{\mathrm{trial}}, \boldsymbol{\xi}_{n+1}^{\text {trial }}, \boldsymbol{\xi}_{\mathrm{d}, n+1}^{\text {trial }}\right\}:=\{\boldsymbol{I}, \mathbf{0}, \mathbf{0}\} .
$$

In correspondence with this trial state, under continuous deformation at a certain stage either nucleation of damage in the austenite occurs or a martensitic transformation is initiated. In order to nucleate damage in the austenite, the trial state damage driving force evaluated at the ambient temperature $\theta^{*}, g_{n+1}^{(0) \text {, trial }}=\left(\mathbb{C}^{\mathrm{A}} \boldsymbol{E}_{n+1} \cdot \boldsymbol{E}_{n+1}\right) / 2+g_{\text {th }}^{(\alpha)}\left(\theta^{*}\right)$, needs to be larger than its critical value, i.e. $g_{n+1}^{(0), \text { trial }}>g_{\mathrm{cr}}^{(0)}$. If this is true, the Newton-Raphson procedure, equation (17), is activated until the residual equations, equations (19), provide a converged solution for the damaged volume fraction of austenite, $\xi_{\mathrm{d}, n+1}^{(0)}$ and the other primary variables. Subsequently, the vector with the damaged volume fractions $\boldsymbol{\xi}_{\mathrm{d}, n+1}$ is updated with the value $\xi_{\mathrm{d}, n+1}^{(0)}$, such that for the next incremental step the trial state (related to a fully austenitic material with damage) becomes

$$
\left\{\boldsymbol{F}_{\mathrm{tr}, n+1}^{\mathrm{trial}}, \boldsymbol{\xi}_{n+1}^{\text {trial }}, \boldsymbol{\xi}_{\mathrm{d}, n+1}^{\text {trial }}\right\}:=\left\{\boldsymbol{I}, \mathbf{0}, \boldsymbol{\xi}_{\mathrm{d}, n}\right\}
$$

with the components $\xi_{\mathrm{d}, n}^{(\alpha)}$ of the vector $\boldsymbol{\xi}_{\mathrm{d}, n}$ equal to zero for $\alpha=1,2, \ldots, N$ (martensite) and non-zero for $\alpha=0$ (austenite).

Alternatively, a martensitic transformation may be activated if for one or more transformation systems the transformation driving force in the trial state exceeds the corresponding critical transformation barrier, i.e.

$$
f_{n+1}^{(\alpha), \text { trial }}>f_{\mathrm{cr}}^{(\alpha)}, \quad \text { where } \alpha \in\{1,2, \ldots, N\} .
$$

The trial state transformation driving forces can be computed substituting the trial state conditions, equation (26) (if there is no damage in the austenite) or equation (27) (if there is damage in the austenite), into equation $(22)_{1}$, which, together with equation $(23)_{1-2}$, leads to

$f_{n+1}^{(\alpha), \text { trial }}=\boldsymbol{F}_{n+1}^{\mathrm{T}} \boldsymbol{F}_{n+1} \mathbb{C}_{*}^{\mathrm{A}} \boldsymbol{E}_{n+1} \cdot \gamma^{(\boldsymbol{\alpha})}+\frac{1}{2}\left(\mathbb{C}^{\mathrm{A}}-\left(1+\delta_{\mathrm{T}}\right) \mathbb{C}^{(\alpha)}\right) \boldsymbol{E}_{n+1} \cdot \boldsymbol{E}_{n+1}+f_{\mathrm{th}}^{(\alpha)}-\frac{\chi}{l_{0}}$,

where $\mathbb{C}_{*}^{\mathrm{A}}=\mathbb{C}^{\mathrm{A}}$ when there is no damage in the austenite and $\mathbb{C}_{*}^{\mathrm{A}}=\left(1-\xi_{\mathrm{d}, n}^{(0)}\right) \mathbb{C}^{\mathrm{A}}$ when the austenitic phase is damaged.

\subsection{Constraints on volume fractions and damaged volume fractions}

If, for a specific martensitic transformation system, the condition given by equation (28) is fulfilled, that system is considered to be active and added to the trial set of active transformation systems, $\mathcal{L}_{\text {trial }}=\left\{\alpha \mid f_{n+1}^{(\alpha) \text {,trial }}>f_{\text {cr }}^{(\alpha)}, \alpha=1,2, \ldots N\right\}$. It is emphasised that $\mathcal{L}_{\text {trial }}$ only may be used in Newton-Raphson iterations related to a fully austenitic material, since the system of active transformation systems generally changes with deformation and therefore needs to be updated continuously. The update of the set of active transformation systems is performed using the search algorithm described in [19]. This search algorithm is based upon algorithms proposed for the numerical modelling of crystal plasticity [20,21], as motivated from the analogy between slip systems and transformation systems. During the transformation process the set of active systems at the onset of a new step $t_{n+1}$ is prescribed to be equal to the set of active systems at the last converged step $t_{n}$, i.e. $\mathcal{L}_{n+1}=\mathcal{L}_{n}$. With this initial set of transformation systems and the trial state given by equation (25), the Newton-Raphson procedure, equation (17), is executed until convergence is reached. In the converged state it 
is first checked if the incremental volume fractions are positive for all transformation systems present in the set of active systems:

$$
\Delta \xi_{n+1}^{(\alpha)}>0, \quad \forall \alpha \in \mathcal{L}_{n+1} .
$$

Since negative volume fractions do not have a physical meaning for irreversible phase transformations, they should be excluded. Hence, if the condition given by equation (30) is violated, the system with the most negative volume fraction is removed from the set of active systems $\mathcal{L}_{n+1}$ and the iterative procedure is restarted. This procedure is repeated until all transformation systems present in $\mathcal{L}_{n+1}$ relate to a positive incremental volume fraction in the converged state. Subsequently, it is checked in the converged state whether additional transformation systems have become active that are not included in the set of active transformation systems, i.e. the condition

$$
f_{n+1}^{(\alpha)}<f_{\text {cr }}^{(\alpha)}, \quad \forall \alpha \notin \mathcal{L}_{n+1},
$$

should hold. If this is not the case, the most loaded transformation system that satisfies the criterion $f_{n+1}^{(\alpha)}>f_{\mathrm{cr}}^{(\alpha)}$ is added to the set of active transformation systems, and the iterative procedure is restarted. This process is repeated until in the converged state all systems not present in the set of active systems $\mathcal{L}_{n+1}$ have a transformation driving force smaller than their critical value $f_{\mathrm{cr}}^{(\alpha)}$.

The checks given by equations (30) and (31) are performed by means of two nested loops, where equation (30) reflects the inner loop and equation (31) represents the outer loop. However, if there is crystalline damage generated in the martensite, the above search algorithm needs to be extended with two additional loops in which the constraints on the damaged volume fractions $\xi_{\mathrm{d}, n+1}$ are checked. A martensitic transformation system will be subjected to damage if the corresponding driving force $g_{n+1}^{(\alpha)}$ given by equation $(22)_{2}$ exceeds the critical threshold value $g_{\mathrm{cr}}^{(\alpha)}$. Subsequently, the Newton-Raphson algorithm determines the corresponding incremental damaged volume fraction $\Delta \xi_{\mathrm{d}, n+1}^{(\alpha)}$ through equation (19)2. In the converged state, the first additional loop related to damage development checks if for all martensitic transformation systems the maximum allowable damaged volume fraction is not exceeded, i.e.

$$
\xi_{\mathrm{d}, n+1}^{(\alpha)} \leqslant \xi_{n+1}^{(\alpha)}, \quad \forall \alpha \in\{1,2, \ldots, N\} .
$$

If this condition is not satisfied, the system with the maximal damage overshoot, $\xi_{\mathrm{d}, n+1}^{(\alpha)}-\xi_{n+1}^{(\alpha)}$, is incorporated in a set $\mathcal{L}_{\mathrm{d}, n+1}$ of maximally damaged martensitic transformation systems. Subsequently the iterative procedure is restarted, where for the systems included in $\mathcal{L}_{\mathrm{d}, n+1}$ the residual in equation $(19)_{2}$ is replaced by

$$
\boldsymbol{r}_{n+1}^{\mathrm{II}}:=\Delta \boldsymbol{\xi}_{\mathrm{d}, n+1}+\boldsymbol{\xi}_{\mathrm{d}, n}-\boldsymbol{\xi}_{n+1},
$$

which essentially reflects the following condition for a maximally damaged phase

$$
\xi_{\mathrm{d}, n+1}^{(\alpha)}=\left(\xi_{\mathrm{d}, n+1}^{(\alpha)}\right)_{\max }=\xi_{n+1}^{(\alpha)}, \quad \forall \alpha \in \mathcal{L}_{\mathrm{d}, n+1} .
$$

The above process is repeated until for all martensitic transformation systems equation (32) is satisfied in the converged state. Subsequently, by means of a second additional loop it is checked for the systems incorporated in $\mathcal{L}_{\mathrm{d}, n+1}$ whether the updated damaged volume fraction related to equation (33) is smaller than the damaged volume fraction following from equation $(19)_{2}$. This requirement can be expressed through the condition

$$
\xi_{n+1}^{(\alpha)}-\xi_{\mathrm{d}, n}^{(\alpha)}<\zeta_{\mathrm{d}, n+1}^{(\alpha)} \Delta t, \quad \forall \alpha \in \mathcal{L}_{\mathrm{d}, n+1} .
$$

If equation (35) is violated for a specific system $\alpha^{\#}$, this system should no longer be present in the set of maximally damaged transformation systems, $\mathcal{L}_{\mathrm{d}, n+1}$, since the kinetic law for 
crystalline damage, equation $(19)_{2}$, then predicts that the damaged volume fraction $\xi_{\mathrm{d}, n+1}^{\left(\alpha^{\#}\right)}$ is again less than the corresponding volume fraction $\xi_{n+1}^{\left(\alpha^{\#}\right)}$. When more than one transformation system violates the criterion in equation (35), the system $\alpha$ with the smallest value for $\zeta_{\mathrm{d}, n+1}^{(\alpha)}$ is removed from $\mathcal{L}_{\mathrm{d}, n+1}$, and the iterative procedure, equation (17), is re-initiated. This process is repeated until all systems included in $\mathcal{L}_{\mathrm{d}, n+1}$ meet the requirement given by equation (35). In summary, in terms of the state variables $\xi_{n+1}^{(\alpha)}$ and $\xi_{\mathrm{d}, n+1}^{(\alpha)}$ of the martensitic transformation systems, the converged solution is found acceptable if the four nested loops represented by equations (30) (= innermost loop), (31), (32) and (35) (= outermost loop) are satisfied.

It should be mentioned that the specific form of the damage kinetic law, equation (10), automatically satisfies the condition in equation (32), since for this law in the course of deformation the damaged volume fraction $\xi_{\mathrm{d}}^{(\alpha)}$ evolves asymptotically to the current value of the volume fraction $\xi^{(\alpha)}$. However, for alternative damage kinetic laws, for example those laws where the rate of the damaged volume fraction $\dot{\xi}_{\mathrm{d}}^{(\alpha)}$ only depends on the damage driving force $g^{(\alpha)}$, the requirement given by equation (32) in principle can be violated during the iterative update procedure. Additional computations (not shown here) have demonstrated that for such kinetic laws the above search algorithm provides a numerical result where all constraints on $\xi^{(\alpha)}$ and $\xi_{\mathrm{d}}^{(\alpha)}$ are satisfied accurately.

In addition to the above constraints, at the beginning of every new iteration $k+1$ of the Newton-Raphson process, it is required that the incremental damaged volume fractions of the austenite and all martensitic transformation systems are non-negative, i.e.

$$
\Delta \xi_{\mathrm{d}, n+1}^{(\alpha), k+1}=\max \left(\Delta \xi_{\mathrm{d}, n+1}^{(\alpha), k+1}, 0\right), \quad \forall \alpha \in\{0,1, \ldots, N\} .
$$

This constraint prescribes that damage can only increase or remain constant and that 'healing' of the material (which corresponds to $\Delta \xi_{\mathrm{d}, n+1}^{(\alpha)}<0$ ) is excluded. Furthermore, a constraint related to the total number of transformation systems is formulated. This constraint accounts for the fact that the transformation process is considered to be completed when the volume fraction of austenite, $\xi_{n+1}^{(0)}$, has either (i) fully transformed, (ii) fully damaged or (iii) fully transformed and damaged. In correspondence with equation $(8)_{1}$, this constraint is formulated as

$$
\xi_{\mathrm{d}, n+1}^{(0)}+\sum_{\alpha=1}^{N} \xi_{n+1}^{(\alpha)}=1
$$

with $\xi_{\mathrm{d}, n+1}^{(0)}$ the damaged volume fraction of austenite. In the Newton-Raphson procedure, equation (37) is satisfied in an approximate manner, through checking in the converged state if

$$
1 \leqslant \xi_{\mathrm{d}, n+1}^{(0)}+\sum_{\alpha=1}^{N} \xi_{n+1}^{(\alpha)}<1+\varepsilon_{\mathrm{m}}
$$

where $\varepsilon_{\mathrm{m}}=10^{-3}$ is a small tolerance. When equation (38) is met, the transformation process is considered to be finished. Conversely, when $\xi_{\mathrm{d}, n+1}^{(\alpha)}+\sum_{\alpha=1}^{N} \xi_{n+1}^{(\alpha)} \geqslant 1+\varepsilon_{\mathrm{m}}$, the incremental step size of the update procedure is considered to be too large and is decreased using a substepping algorithm. In the substepping algorithm, the step size represented by the incremental total deformation gradient, equation (15), is divided into smaller steps by means of a bisection procedure. The reduction in step size is continued until a step size is reached for which equation (38) is satisfied, see [19] for more details.

If, at a certain prescribed minimum step size, the iterative procedure still does not converge, the set of active transformation systems $\mathcal{L}_{n+1}$ is emptied, and a new set of active transformation system is constructed using the procedure outlined by equations (30) and (31). Apart from 
being used for satisfying the transformation criterion equation (38), the substepping algorithm is also used to provide an accurate incremental update for integration points subjected to complex changes in transformation and damage activities, where relatively small integration steps are required to obtain a properly converged numerical solution. More details on the substepping algorithm can be found in [19].

\subsection{Stress update and tangent operator}

After the iterative procedure, equation (17), has converged, the finite element program ABAQUS requires the updated Cauchy stress $\boldsymbol{T}_{n+1}$ in order to construct the element residual vector at the global (system) level. The updated Cauchy stress can be computed from the updated second Piola-Kirchhoff stress in the intermediate configuration, $\boldsymbol{S}_{n+1}$. The values of the components of $\boldsymbol{S}_{n+1}$ follow from the update of the essential variable $\boldsymbol{a}_{n+1}^{\mathrm{IV}}$, see equation (18) 4 . The stresses $\boldsymbol{S}_{n+1}$ and $\boldsymbol{T}_{n+1}$ are connected through the expression

$$
\boldsymbol{T}_{n+1}=\left(\operatorname{det} \boldsymbol{F}_{\mathrm{e}, n+1}\right)^{-1} \boldsymbol{F}_{\mathrm{e}, n+1} \boldsymbol{S}_{n+1} \boldsymbol{F}_{\mathrm{e}, n+1}^{\mathrm{T}},
$$

with the updated elastic deformation gradient $\boldsymbol{F}_{\mathrm{e}, n+1}$ given by equation $(24)_{2}$. In order to construct the global stiffness matrix at the system level, ABAQUS requires the calculation of a tangent operator in the Eulerian setting, which connects the velocity gradient to the Jaumann derivative of the Kirchhoff stress. This tangent operator is constructed by first computing a tangent operator in the Lagrangian setting, which relates to the first Piola-Kirchhoff stress in the reference configuration,

$$
\boldsymbol{P}_{n+1}=\operatorname{det}\left(\boldsymbol{F}_{\mathrm{tr}, n+1}\right) \boldsymbol{F}_{n+1} \boldsymbol{F}_{\mathrm{tr}, n+1}^{-1} \boldsymbol{S}_{n+1} \boldsymbol{F}_{\mathrm{tr}, n+1}^{-T},
$$

and the total deformation gradient $\boldsymbol{F}_{n+1}$. This tangent operator is computed numerically from perturbations of $\boldsymbol{F}_{n+1}$ and $\boldsymbol{P}_{n+1}$, using a first-order accurate forward finite-difference scheme. Subsequently, the Lagrangian tangent operator is transformed to the above-mentioned Eulerian tangent operator through the application of appropriate transformation rules. More details on the computation of the tangent operator can be found in [19].

\section{Numerical simulations}

\subsection{Microstructural geometry, boundary conditions and constitutive parameters}

The microstructural sample considered in the numerical analyses is depicted in figure 3 , and consists of a grain of retained austenite $\Omega^{\mathrm{gr}}$ embedded in a ferrite-based matrix $\Omega^{\mathrm{mat}}$. This sample is representative of the phases present in a high-strength TRIP steel. The local carbon concentration in the retained austenite is taken as $1.4 \mathrm{wt} . \%$. The sample has a cuboidal shape and occupies a domain $\Omega=\Omega^{\mathrm{gr}} \cup \Omega^{\text {mat }}$ equal to $3 L \times 3 L \times 3 L$, where $3 L$ is the length of the sides of the cube. The austenite grain has a polyhedral shape and in the undeformed state occupies about $16 \%$ of the total domain $\Omega$. The length $L$ is taken equal to $1 \mu \mathrm{m}$, which corresponds to an average size of the austenitic grain of approximately $2 \mu \mathrm{m}$. The initial austenitic volume fraction and the size of the austenite grain are chosen in accordance with typical experimental values related to multiphase carbon steels [1,22-24]. The cubic sample is discretized using 1771 linear tetrahedron elements, of which 314 elements are used to model the austenite grain and 1457 elements are used to model the ferrite-based matrix.

The boundary conditions applied to the sample are as follows. In the directions normal to three of the six cube faces the displacement is set to zero and the top surface of the cube is loaded in the axial direction $\left(=f_{1}\right.$-direction) through applying a displacement $u_{1}(t)=(3 L \varepsilon) /(t / T)$, with $\varepsilon=0.14$. The tangential stresses on these four faces are zero, and the two remaining faces 


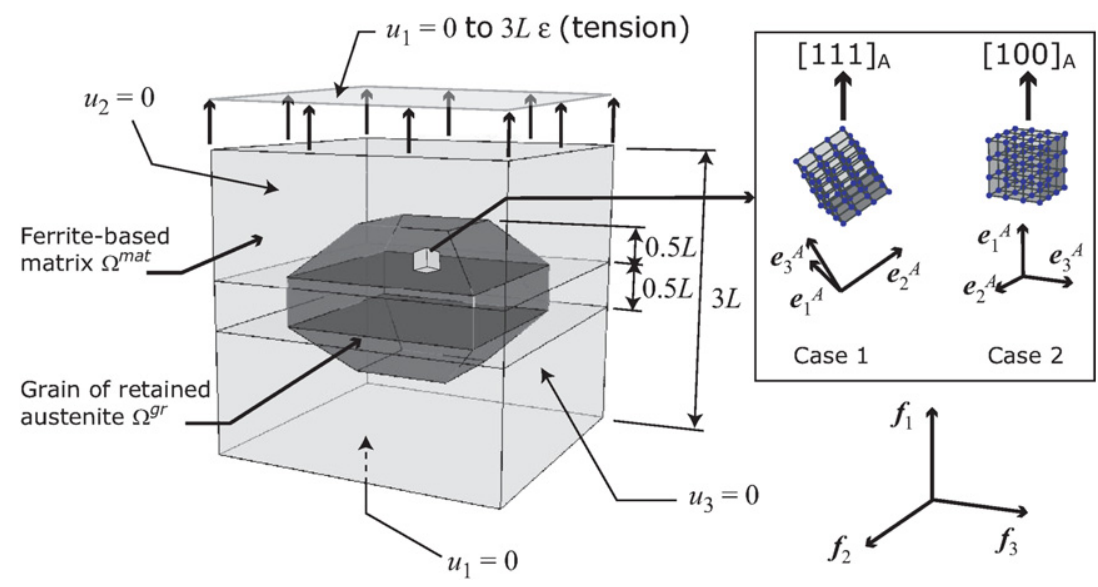

Figure 3. Domain of a single grain of retained austenite $\Omega^{\mathrm{gr}}$ embedded in a ferrite-based matrix $\Omega^{\text {mat }}$. The inset shows the considered lattice orientations, $\left[\begin{array}{lll}1 & 1 & 1\end{array}\right]_{\mathrm{A}}$ and $\left[\begin{array}{lll}1 & 0 & 0\end{array}\right]_{\mathrm{A}}$, for a material point inside the grain, which are expressed in terms of the lattice vectors of the austenite grain, $\left\{e_{1}^{\mathrm{A}}, e_{2}^{\mathrm{A}}, e_{3}^{\mathrm{A}}\right\}$. The global basis is indicated by the vectors $\left\{f_{1}, f_{2}, f_{3}\right\}$, where $f_{1}$ represents the loading direction.

(This figure is in colour only in the electronic version)

are stress free. The total analysis time is set to $T=1400 \mathrm{~s}$ which corresponds to a nominal, quasi-static axial strain rate of $10^{-4} \mathrm{~s}$. The analyses are performed under isothermal conditions, at an ambient temperature of $300 \mathrm{~K}$. Two different crystal orientations are considered, where the uniaxial loading directions are $\left[\begin{array}{lll}1 & 0 & 0\end{array}\right]_{\mathrm{A}}$ and $\left[\begin{array}{lll}1 & 1 & 1\end{array}\right]_{\mathrm{A}}$, with the index $\mathrm{A}$ indicating that the direction is measured in the austenite lattice basis $\left\{\boldsymbol{e}_{1}^{\mathrm{A}}, \boldsymbol{e}_{2}^{\mathrm{A}}, \boldsymbol{e}_{3}^{\mathrm{A}}\right\}$.

The material parameters for the phase-changing damage model are summarized in table 1 and are representative of retained austenite in high-strength TRIP steels. In TRIP steels the martensitic product phase is typically much more brittle than the austenitic parent phase; tensile tests performed on TRIP-assisted multiphase steels show that premature crystalline damage occurs mainly in the martensitic phase [7,9]. In the present simulations it is therefore assumed that damage may only become active in the martensitic transformation systems $\alpha=1,2, \ldots, N$. The damage in the austenite is prevented adopting a relatively high value for the austenite damage threshold $g_{\mathrm{cr}}^{(0)}$. More details on the calibration of the material parameters for the elastic and phase-transformation behaviour can be found in [25] and for damage behaviour in [10].

The elasto-plastic response of the ferrite-based matrix is simulated by a $J_{2}$-plasticity model with isotropic hardening, formulated within a large deformation framework. Previous computations for an austenitic grain embedded in a ferritic matrix composed of six grains with orientations provided by equally-spaced Euler angles have shown that a BCC crystal plasticity model for the ferritic grains provides a similar uniaxial tensile response as a $J_{2}$-plasticity model [26]. From this result, and the goal of keeping the computational time within reasonable bounds, in the present study it was decided to use a $J_{2}$-plasticity model for the ferritic matrix. Young's modulus and Poisson's ratio of the matrix material are equal to $E=149 \mathrm{MPa}$ and $v=$ 0.35 , respectively. The hardening behaviour of the ferrite-based matrix is modelled through an exponentially saturating hardening law, with an initial yield strength of $300 \mathrm{MPa}$ and a final yield strength in the saturation state of $880 \mathrm{MPa}$. These parameters follow from calibrating the experimental data presented in [1] for a dual phase polycrystalline sample of $75 \mathrm{wt} . \%$ ferrite and $25 \mathrm{wt} . \%$ thermal martensite that was loaded under uniaxial tension, see also [25]. 
Table 1. Values of the material parameters in the phase-changing damage model.

\begin{tabular}{|c|c|c|}
\hline Parameter(s) & Value(s) & Equation(s) \\
\hline $\begin{array}{l}\text { Elastic moduli austenite } \\
\text { to construct stiffness tensor } \mathbb{C}^{\mathrm{A}}\end{array}$ & $\kappa_{1}^{\mathrm{A}}=286.8, \kappa_{2}^{\mathrm{A}}=166.4, \kappa_{3}^{\mathrm{A}}=145.0(\mathrm{GPa})$ & Equation $(5)_{1}$ \\
\hline $\begin{array}{l}\text { Elastic moduli martensite } \\
\text { to construct stiffness tensor } \mathbb{C}^{(\alpha)}\end{array}$ & $\begin{array}{l}\kappa_{1}^{\mathrm{M}}=372.4, \kappa_{2}^{\mathrm{M}}=345.0, \kappa_{3}^{\mathrm{M}}=191.0 \\
\kappa_{4}^{\mathrm{M}}=508.4, \kappa_{5}^{\mathrm{M}}=201.9, \kappa_{6}^{\mathrm{M}}=229.5(\mathrm{GPa})\end{array}$ & Equation $(5)_{1}$ \\
\hline Shape strain magnitude & $\gamma_{\mathrm{T}}=0.1809[-]$ & Equation (3) \\
\hline Transformation dilation & $\delta_{\mathrm{T}}=0.0391[-]$ & Equation (6) \\
\hline Transformation kinetic law & $\begin{array}{l}\dot{\xi}_{\max }^{(\alpha)}=\dot{\xi}_{\max }=3 \times 10^{-3}\left(\mathrm{~s}^{-1}\right) \\
f_{\mathrm{cr}}^{(\alpha)}=f_{\mathrm{cr}}=293(\mathrm{MPa}) \\
v^{(\alpha)}=v=0.17[-]\end{array}$ & Equation (9) \\
\hline $\begin{array}{l}\text { Thermal contribution to } \\
\text { transformation driving force } \\
\text { (isothermal process) }\end{array}$ & $f_{\mathrm{th}}^{(\alpha)}=f_{\mathrm{th}}=288(\mathrm{MPa})$ & Equation $(13)_{1}$ \\
\hline $\begin{array}{l}\text { Surface energy } \\
\text { at habit plane }\end{array}$ & $\begin{array}{l}\chi=0.2 \mathrm{Jm}^{-2} \\
l_{0}=0.05 \mu \mathrm{m}\end{array}$ & Equations $(14)_{3,6}$ \\
\hline Damage kinetic law & $\dot{\xi}_{\mathrm{d}, \max }^{(\alpha)}=\dot{\xi}_{\mathrm{d}, \max }=1 \times 10^{-2}\left(\mathrm{~s}^{-1}\right)$ & \\
\hline martensite & $\begin{array}{l}g_{\mathrm{cr}}^{(\alpha)}=g_{\mathrm{cr}}=220(\mathrm{MPa}) \\
\beta_{\mathrm{d}}^{(\alpha)}=\beta_{\mathrm{d}}=95[-]\end{array}$ & Equation (10) \\
\hline $\begin{array}{l}\text { Thermal contribution to } \\
\text { damage driving force } \\
\text { (isothermal process) }\end{array}$ & $g_{\mathrm{th}}^{(\alpha)}=g_{\mathrm{th}}=213(\mathrm{MPa})$ & Equation $(13)_{2}$ \\
\hline
\end{tabular}

\subsection{Discussion of results}

Figure 4 shows the overall axial Cauchy stress $\bar{T}_{11}$ versus the overall axial logarithmic strain $\bar{e}_{11}$, both averaged over the sample. For the two crystal orientations $\left[\begin{array}{lll}1 & 0 & 0\end{array}\right]_{\mathrm{A}}$ and $\left[\begin{array}{lll}1 & 1 & 1\end{array}\right]_{\mathrm{A}}$, the stressstrain response is plotted for the cases where (i) only a martensitic transformation occurs and crystalline damage is prevented (dashed lines) and (ii) both a martensitic transformation occurs and crystalline damage develops (solid lines). For comparison, the stress-strain response where the whole sample is occupied by the ferritic matrix is also depicted. At the onset of loading, the grain of retained austenite and the ferritic matrix respond elastically. However, when the axial Cauchy stress $\bar{T}_{11}$ is within the range between 250 and $350 \mathrm{MPa}$, transformation of the austenite and plasticity in the ferrite-based matrix are initiated, both for the sample with the $[100]_{\mathrm{A}^{-}}$ loaded grain and for the sample with the $\left[\begin{array}{lll}1 & 1 & 1\end{array}\right]_{\mathrm{A}}$-loaded grain. Subsequently, at a stress value between 700 and $800 \mathrm{MPa}$ damage is initiated in the martensitic product phase, as indicated in figure 4 by the point where the solid and dashed lines separate. When the transformation is completed, the stress-strain responses for the case of transformation without damage (dashed lines) increase monotonically, since the martensitic product phase responds elastically where the stress level grows with increased deformation. In contrast, the responses for the case of transformation with damage (solid lines) show a maximum stress value. The maximum stress for the sample with the $\left[\begin{array}{lll}1 & 0 & 0\end{array}\right]_{\mathrm{A}}$-loaded grain equals $960 \mathrm{MPa}$ and is somewhat higher than the maximum stress for the sample with the $\left[\begin{array}{lll}1 & 1 & 1\end{array}\right]_{\mathrm{A}}$-loaded grain, which is $880 \mathrm{MPa}$. After reaching the maximum stress, the stress decreases and under continuously increasing damage it tends asymptotically to a value that is determined by the plastic response of the ferritic matrix. The asymptotic value of the stress is approximately equal to the saturation strength of the ferritic matrix multiplied by a factor of $0.84(=1-0.16)$ that accounts for the void created by the embedded grain.

In order to analyse the interactions between the transformation and crystalline damage processes in more detail, in figure 5 the martensitic volume fraction $\bar{\xi}_{\mathrm{M}}^{\mathrm{gr}}$ and the damaged 


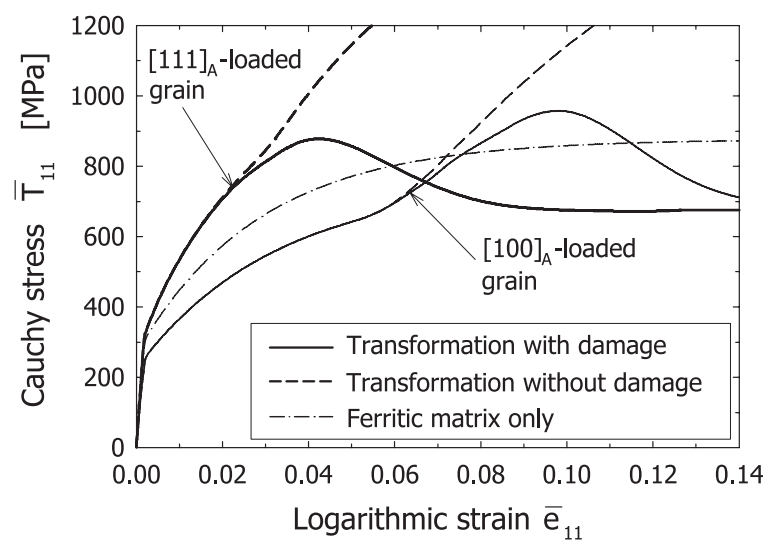

Figure 4. Axial Cauchy stress $\bar{T}_{11}$ versus axial logarithmic strain $\bar{e}_{11}$ for samples with a $\left[\begin{array}{ll}1 & 0\end{array}\right]_{\mathrm{A}}$ oriented austenitic grain and a $\left[\begin{array}{llll}1 & 1 & 1\end{array}\right]_{\mathrm{A}}$-oriented austenitic grain that transform with damage (solid lines) and without damage (dashed lines). For comparison, the stress-strain response of the ferritic matrix (dashed-dotted line) is also depicted.

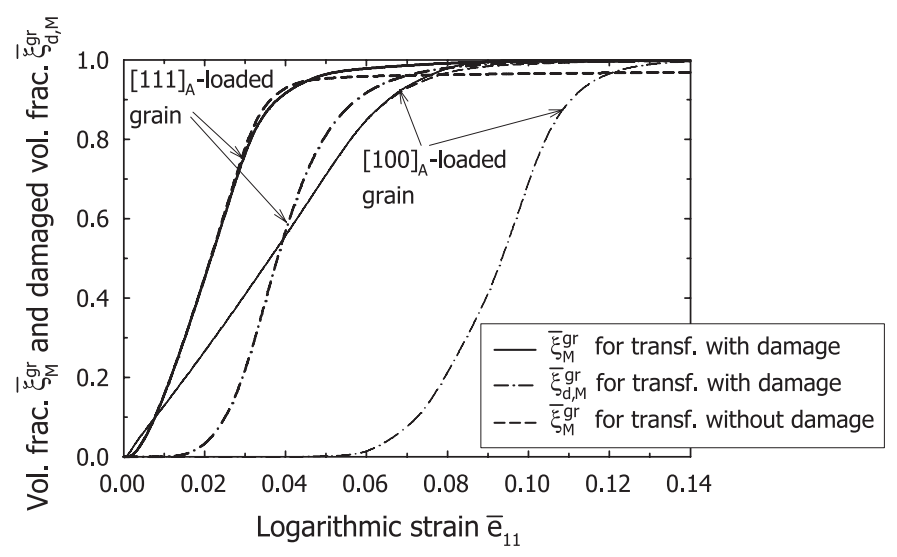

Figure 5. Martensitic volume fraction $\bar{\xi}_{\mathrm{M}}^{\mathrm{gr}}$ and damaged volume fraction $\bar{\xi}_{\mathrm{d}, \mathrm{M}}^{\mathrm{gr}}$ versus axial logarithmic strain $\bar{e}_{11}$ for samples with a $\left[\begin{array}{lll}1 & 0 & 0\end{array}\right]_{\mathrm{A}}$-oriented austenitic grain and a [ $\left[\begin{array}{llll}1 & 1 & 1\end{array}\right]_{\mathrm{A}}$-oriented austenitic grain that transform with damage (solid lines for $\bar{\xi}_{\mathrm{M}}^{\mathrm{gr}}$, dashed-dotted lines for $\bar{\xi}_{\mathrm{d}, \mathrm{M}}^{\mathrm{gr}}$ ) and without damage (dashed lines for $\bar{\xi}_{\mathrm{M}}^{\mathrm{gr}}$ ).

martensitic volume fraction $\bar{\xi}_{\mathrm{d}, \mathrm{M}}^{\mathrm{gr}}$, averaged over the initial volume of the grain, are depicted versus the average axial logarithmic strain $\bar{e}_{11}$. For comparison, the evolution of the volume fraction $\bar{\xi}_{\mathrm{M}}^{\mathrm{gr}}$ for the case where transformation occurs without damage is also plotted. It can be observed that for both grain orientations the evolution of $\bar{\xi}_{\mathrm{M}}^{\mathrm{gr}}$ is only slightly affected by the generation of crystalline damage and that the rate of transformation initially is relatively high, but strongly decreases near completion of transformation. The latter trend has also been observed in tensile experiments on TRIP steels [1,27]. In addition, the martensitic transformation for the sample with the $\left[\begin{array}{lll}1 & 1 & 1\end{array}\right]_{\mathrm{A}}$-loaded grain occurs relatively fast, whereas for the sample with the $\left[\begin{array}{lll}1 & 0 & 0\end{array}\right]_{\mathrm{A}}$-loaded grain it occurs relatively slow. Correspondingly, during the initial stage of deformation the effective strength of the sample with the $\left[\begin{array}{lll}1 & 1 & 1\end{array}\right]_{\mathrm{A}}$-loaded grain is higher than that of ferrite-based matrix, see figure 4, since the generation of a relatively stiff martensitic product phase increases the average stress level in the sample. This effect is 
Table 2. Number of elements for three different mesh densities.

\begin{tabular}{lccc}
\hline Mesh & $\begin{array}{l}\text { Number of elements } \\
\text { austenite }\end{array}$ & $\begin{array}{l}\text { Number of elements } \\
\text { ferrite-based matrix }\end{array}$ & $\begin{array}{l}\text { Total number of } \\
\text { elements }\end{array}$ \\
\hline (1) Coarse & 56 & 228 & 284 \\
(2) Intermediate & 314 & 1457 & 1771 \\
(3) Fine & 1674 & 3656 & 5330 \\
\hline
\end{tabular}

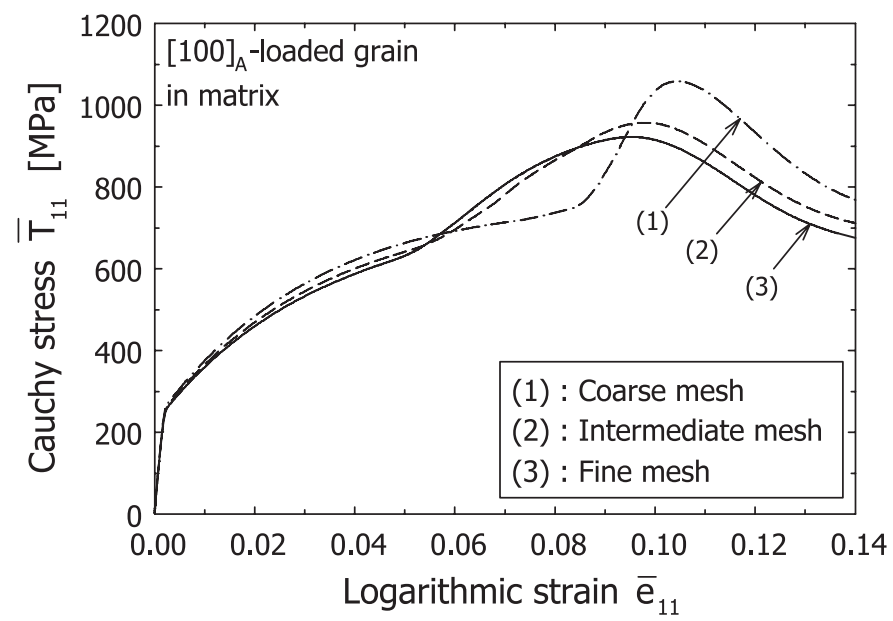

Figure 6. Axial Cauchy stress $\bar{T}_{11}$ versus axial logarithmic strain $\bar{e}_{11}$ for a sample with a $\left[\begin{array}{lll}1 & 0 & 0\end{array}\right]_{\mathrm{A}^{-}}$ oriented austenitic grain that transforms with damage. Three mesh densities are considered: a coarse mesh (dashed-dotted line), an intermediate mesh (dashed line) and a fine mesh (solid line).

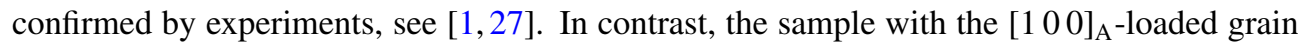
initially has a lower strength than the ferrite-based matrix, since the effective transformation strain is considerably larger in that orientation [25]. In other words, for the $\left[\begin{array}{ll}1 & 0\end{array}\right]_{\mathrm{A}}$ crystal orientation the presence of retained austenite has a pernicious influence on the initial effective strength of the sample. The fact that $\left[\begin{array}{lll}1 & 1 & 1\end{array}\right]_{\mathrm{A}}$ austenite grains and $\left[\begin{array}{lll}1 & 0 & 0\end{array}\right]_{\mathrm{A}}$ austenite grains, respectively, reflect 'strong' and 'weak' orientations in terms of their transformation behaviour is in agreement with experimental observations [28, 29].

In addition, for both crystal orientations it can be observed that crystalline damage in the martensite starts at a deformation stage where the transformation process has not been completed. Although for the $\left[\begin{array}{lll}1 & 1 & 1\end{array}\right]_{\mathrm{A}}$-loaded grain the damage starts at a much earlier stage than for the $\left[\begin{array}{lll}1 & 0 & 0\end{array}\right]_{\mathrm{A}}$-loaded grain, the rate of damage is similar for both crystal orientations. Under continuous deformation the damaged martensitic volume fraction $\bar{\xi}_{\mathrm{d}, \mathrm{M}}^{\mathrm{gr}}$ closely approaches the corresponding martensitic volume fraction $\bar{\xi}_{\mathrm{M}}^{\mathrm{gr}}$, which implies that the martensite then is almost completely damaged.

\subsection{Mesh refinement study}

In order to analyse the convergence behaviour of the numerical solution upon mesh refinement, the boundary value problem for the sample shown in figure 3 is performed for three different meshes, namely (i) a coarse mesh, (ii) an intermediate mesh and (iii) a fine mesh.

The number of elements for each of these meshes is presented in table 2. Note that the intermediate mesh represents the mesh that was used for the simulations discussed in 


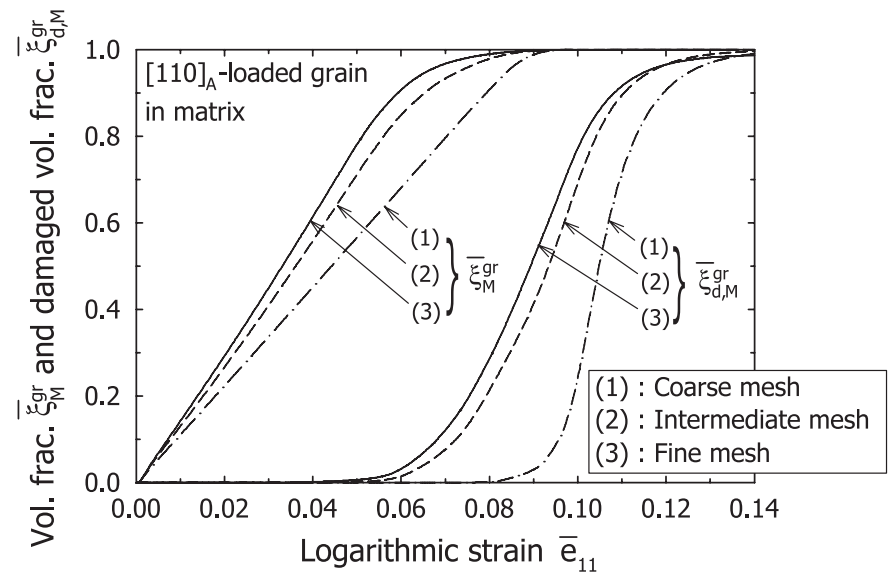

Figure 7. Martensitic volume fraction $\bar{\xi}_{\mathrm{M}}^{\mathrm{gr}}$ and damaged volume fraction $\bar{\xi}_{\mathrm{d}, \mathrm{M}}^{\mathrm{gr}}$ versus axial logarithmic strain $\bar{e}_{11}$ for a sample with a $\left[\begin{array}{lll}1 & 0 & 0\end{array}\right]_{\mathrm{A}}$-oriented austenitic grain that transforms with damage. Three mesh densities are considered: a coarse mesh (dashed-dotted line), an intermediate mesh (dashed line) and a fine mesh (solid line).

sections 4.1 and 4.2. The increase in mesh density from the coarse mesh to the intermediate mesh is approximately a factor of 6 and from the intermediate mesh to the fine mesh is about a factor of 3 .

The stress-strain responses related to the three different meshes are shown in figure 6 for the sample with the $\left[\begin{array}{lll}1 & 0 & 0\end{array}\right]_{\mathrm{A}}$-loaded grain. It can be observed that the response for the coarse mesh is significantly different from the responses for the intermediate and fine meshes, especially at larger deformations where crystalline damage in the martensite becomes significant. The coarse mesh not only seriously overpredicts the peak strength, but also extends the deformation range during which the transformation takes place. This can be seen more clearly in figure 7 , which shows the evolution of the martensitic volume fraction $\bar{\xi}_{\mathrm{M}}^{\mathrm{gr}}$ and the damaged martensitic volume fraction $\bar{\xi}_{\mathrm{d}, \mathrm{M}}^{\mathrm{gr}}$ for the three different meshes. The onset of damage for the coarse mesh occurs at larger deformation than for the intermediate and fine meshes. Because both the stress-strain response and the transformation and damage evolutions do not differ much for the intermediate and fine meshes, it is concluded that the results presented in section 4.2 are sufficiently accurate.

\section{Conclusions}

A numerical update algorithm has been provided for the phase-changing damage model presented in Suiker and Turteltaub [10]. The ability of the algorithm to solve problems where complex small-scale interactions between damage, transformation and plasticity processes play a role is demonstrated through three-dimensional analyses of a TRIP steel microstructure. The simulated microstructural sample consists of a single grain of retained austenite embedded in a ferritic matrix, which is subjected to uniaxial tension. A variation study of the density of the finite element mesh indicates that the numerical results tend to converge upon mesh refinement.

The numerical simulations illustrate that the development of crystalline damage in the martensitic product phase may significantly limit the stresses developing in the grain. A comparison between samples with a $\left[\begin{array}{lll}1 & 0 & 0\end{array}\right]_{A}$-loaded austenite grain and a $\left[\begin{array}{lll}1 & 1 & 1\end{array}\right]_{A}$-loaded austenite grain reveals that both the onset of damage and the maximum stress in the transforming 
grain depend strongly on the grain orientation. During the initial stage of deformation, the sample with the $\left[\begin{array}{lll}1 & 1 & 1\end{array}\right]_{\mathrm{A}}$-loaded grain has the largest hardening capacity, since for this sample the transformation from austenite to martensite occurs at a higher rate than for the sample with the $\left[\begin{array}{lll}1 & 0 & 0\end{array}\right]_{\mathrm{A}}$-loaded grain. Nonetheless, the largest ultimate strength is obtained for the sample with the $\left[\begin{array}{lll}1 & 0 & 0\end{array}\right]_{A}$-loaded grain. The main trends related to the transformation behaviour of the grain are in agreement with experimental results for TRIP steels reported in the literature.

Although in the present work the numerical results were reported in terms of average values over the grains, the finite element simulations showed that crystalline damage of the martensite nucleates first on the grain boundary and subsequently in the interior of the grain. This sequence of damage events in the simulations, which has also been observed experimentally in the TRIP steel samples tested by Jacques et al [7], can be ascribed to the polyhedral shape of the grain, see figure 3, which induces stress concentrations at the grain boundary with the ferritic matrix.

As a final remark, it is mentioned that the present simulations are representative of TRIP steel microstructures where plasticity in the austenite (which has not been accounted for in the model) is considered as a minor effect. This is the case if the carbon concentration in the austenite is relatively high (i.e. $>1.4 \mathrm{wt} . \%$ ), where the dislocations present in the austenite are pinned by the carbon atoms, and most of the plastic deformation induced by the martensitic transformation occurs in the neighbouring ferritic phase.

\section{Acknowledgments}

This work is part of the research program of the Netherlands Institute for Metals Research (NIMR) and the Stichting voor Fundamenteel Onderzoek der Materie (FOM, financially supported by the Nederlandse Organisatie voor Wetenschappelijk Onderzoek (NWO)). The research was carried out under Project Number 02EMM20 of the FOM/NIMR Program 'Evolution of the Microstructure of Materials' (P-33).

\section{References}

[1] Jacques P J, Furnémont Q, Mertens A, and Delannay F. On the sources of work hardening in multiphase steels assisted by transformation-induced plasticity. Phil. Mag. A 81 1789-812

[2] Evans A G 1990 Perspective on the development of high-toughness ceramics J. Am. Ceram. Soc. 73 187-206

[3] Bhattacharya K 2004 Microstructure of Martensite (Oxford: Oxford University Press)

[4] Chen J H, Kikuta Y, Araki T, Yoneda M and Matsuda Y 1984 Micro-fracture behavior induced by M-A constituent (island martensite) in simulated welding heat-affected zone of HT80 high-strength low alloyed steel Acta Metall. 32 1779-88

[5] Taillard R, Verrier P, Maurickx T and Foct J 1995 Effect of Silicon on CHAZ toughness and microstructure of microalloyed steels Metall. Mater. Trans. A 26 447-57

[6] Nakatsu H and Takaki S 1996 Effect of austenite grain size in Fe-Mn alloys on epsilon martensitic transformation and their mechanical properties. J. Japan Inst. Met. $60141-8$

[7] Jacques P, Furnémont Q, Pardoen T and Delannay F 2001 On the role of martensitic transformation on damage and cracking resistance in TRIP-assisted multiphase steels Acta Mater. 49 139-52

[8] Lu Y H, Qiao L J and Chu W Y 2002 In situ study of martensitic transformation and nucleation and propagation of cracks in $\mathrm{Cu}-\mathrm{Ni}-\mathrm{Al}$ shape memory alloy Mater. Sci. Technol. 18 273-8

[9] Papaefthymiou S 2005 Failure Mechanisms of Multiphase Steels PhD Thesis RWTH Aachen University, Aachen

[10] Suiker A S J and Turteltaub S 2006 Crystalline damage growth during martensitic phase transformations submitted

[11] Wechsler M S, Lieberman D E, and Read T A 1953 On the theory of the formation of martensite Trans. Am. Inst. Min. Metall. Eng. 197 1503-15

[12] Ball J M and James R D 1987 Fine phase mixtures as minimizers of energy Arch. Ration. Mech. Anal. 100 13-52

[13] Lee E H 1969 Elastic-plastic deformation at finite strains J. Appl. Mech. (ASME) 36 1-6 
[14] Hill R and Rice J R 1972 Constitutive analysis of elastic-plastic crystals at arbitrary strain J. Mech. Phys. Solids 20 401-13

[15] Turteltaub S and Suiker A S J 2006 A multiscale thermomechanical model for cubic to tetragonal martensitic phase transformations Int. J. Solids Struct. $\mathbf{4 3} 4509-45$

[16] Hane K F and Shield T W 1998 Symmetry and microstructure in martensites Phil. Mag. A 78 1215-52

[17] Borja R 1991 Cam-clay plasticity, Part II, Implicit integration of constitutive equations based on a nonlinear elastic stress predictor Comput. Meth. Appl. Mech. Eng. 88 225-40

[18] Heeres O M, Suiker A S J and de Borst R 2002 A comparison between the Perzyna viscoplastic model and the Consistency viscoplastic model Eur. J. Mech. A/Solids 21 1-12

[19] Suiker A S J and Turteltaub S 2005 Computational modelling of plasticity induced by martensitic phase transformations Int. J. Numer. Meth. Eng. 63 1655-93

[20] Cuitiño A M and Ortiz M 1993 Computational modelling of single crystals Modell. Simul. Mater. Sci. Eng. $1225-63$

[21] Miehe C and Schröder J 2001 A comparative study of stress update algorithms for rate-independent and ratedependent crystal plasticity Int. J. Numer. Meth. Eng. 50 273-98

[22] Sugimoto K-I, Mitsuyuki K and Hashimoto S-I 1992 Ductility and strain-induced transformation in a highstrength transformation-induced plasticity-aided dual-phase steel Metall. Trans. A 23 3085-91

[23] Furnémont Q, Kempf M, Jacques P J, Göken M and Delannay F 2002 On the measurement of the nanohardness of the constitutive phases of TRIP-assisted multiphase steels. Mater. Sci. Eng. A 328 26-32

[24] Streicher A M, Speer J G and Matlock D K 2002 Forming response of retained austenite in a C-Si-Mn high strength TRIP sheet steel. Steel Res. 73 287-93

[25] Turteltaub S and Suiker A S J 2005 Transformation-induced plasticity in ferrous alloys J. Mech. Phys. Solids 53 1747-88

[26] Tjahjanto D D, Turteltaub S, Suiker A S J and van der Zwaag S 2006 Modelling of the effects of grain orientation on transformation-induced plasticity in multiphase carbon steels Modell. Simul. Mater. Sci. Eng. 14 617-36

[27] Jacques P J, Ladriére J and Delannay F 2001 On the influence of interactions between phases on the mechanical stability of retained austenite in transformation-induced plasticity multiphase steels Metall. Mater. Trans. A 32 2759-68

[28] Oliver E C, Withers P J, Daymond M R, Ueta S and Mori T 2002 Neutron-diffraction study of stress-induced martensitic transformation in TRIP steel Appl. Phys. A - Mater. Sci. Process. 74 S1143-5

[29] Kruijver S O, Zhao L, Sietsma J, Offerman S E, van Dijk N H, Lauridsen E M, Margulies L, Grigull S, Poulsen H F and van der Zwaag S 2003 In situ observations on the mechanical stability of austenite in TRIP-steel J. Physique IV 104 499-502 